\title{
A GENERAL PROCEDURE FOR DERIVING STABILIZED SPACE-TIME FINITE ELEMENT METHODS FOR ADVECTIVE-DIFFUSIVE PROBLEMS
}

\author{
E. OÑATE ${ }^{\mathrm{a}, *}$ AND M. MANZÁN ${ }^{\mathrm{b}}$ \\ ${ }^{a}$ International Center for Numerical Methods in Engineering, Universidad Politécnica de Cataluña, Gran Capitán s/n, \\ 08034 Barcelona, Spain \\ ${ }^{\mathrm{b}}$ University of Udine, Udine, Italy
}

\begin{abstract}
SUMMARY
A procedure to derive stabilized space-time finite element methods for advective-diffusive problems is presented. The starting point is the stabilized balance equation for the transient case derived by Oñate [Comput. Methods Appl. Mech. Eng., 151, 233-267 (1998)] using a finite increment calculus approach. A description of the new stabilization method and a procedure for computing the stabilization parameter of the space-time solution is given. The efficiency of the stabilization approach is shown in the solution of some transient advective-diffusive problems, including the non-linear Burger's equation. Copyright (C) 1999 John Wiley \& Sons, Ltd.
\end{abstract}

KEY WORDS: $\quad$ space-time finite element methods; advective-diffusive problems; stabilization problem

\section{INTRODUCTION}

Space-time formulations are direct time integration methods. The simplest time discontinuous Galerkin method generates a complete space-time finite element discretization, where continuity across the slab interfaces is weakly enforced eliminating, however, the need for an additional ordinary differential equation solver to discretize time. Application of the spacetime (discontinuous) approach to advective-diffusive and fluid flow problems were reported in References [4-15]. An advantage of the space-time formulation is that the deformation of the spatial domain can be taken into account automatically. This feature has been exploited by Tezduyar et al. [11-13] and Masud and Hughes [15] to solve a number of fluid flow problems involving moving boundaries and interfaces.

The time discontinuous Galerkin method, similar to the Galerkin method for the steady case, lacks sufficient stability. A manifestation of this lack of stability is the spurious oscillations generated by unresolved internal and boundary layers. The standard solution to this problem is adding a series of $a d$ hoc stabilization terms to the Galerkin formulation of the problem.

The more popular approach is to choose these stabilization terms by minimizing the sum of the square residual of the momentum equation integrated over each element domain [19]. The

* Correspondence to: International Center for Numerical Methods in Engineering, Universidad Politécnica de Cataluña, Gran Capitán s/n, 08034 Barcelona, Spain.

CCC 0271-2091/99/170203-19\$17.50

Copyright (C) 1999 John Wiley \& Sons, Ltd. 
resulting least-square operator is made proportional to a stabilization coefficient. The precise computation of this crucial coefficient is still an open issue and ad hoc extensions of the values obtained for simple one-dimensional cases are used in practice [10,16-18].

In this paper a conceptually different approach for deriving a stabilized space-time formulation is proposed. Oñate [1] has shown that most stabilized numerical schemes can be naturally derived from the stabilized form of the governing differential equations of the problem. These equations are obtained by involving higher-order balance laws over a finite balance domain using a kind of 'finite increment calculus' (FIC) procedure. The FIC approach has been also exploited by Oñate et al. [2,3] to propose a general iterative scheme for computing the streamline and transverse (cross-wind) stabilization parameters, which can be interpreted as the dimensions of the finite balance domain, in terms of the solution residuals. The new stabilization scheme can be understood as a particular class of adaptive methods, where the numerical solution is progressively enhanced by the iterative computation of the critical streamline and cross-wind stabilization parameters, while keeping the mesh and the approximation unchanged [3]. Examples of application of this stabilization technique to steady advection-diffusion problems involving boundary layers and sharp internal gradients can be found in References [1-3].

The ideas presented in [1-3] are extended here to derive a stabilized space-time finite element formulation. The analogy of the new formulation with a time discontinuous SUPG approach is shown.

An interesting feature of the new space-time formulation here presented is that it provides a straightforward methodology for computing the stabilization parameter as a function of the residuals of the space-time solution.

The layout of the paper is as follows. In the next section the stabilized differential equations

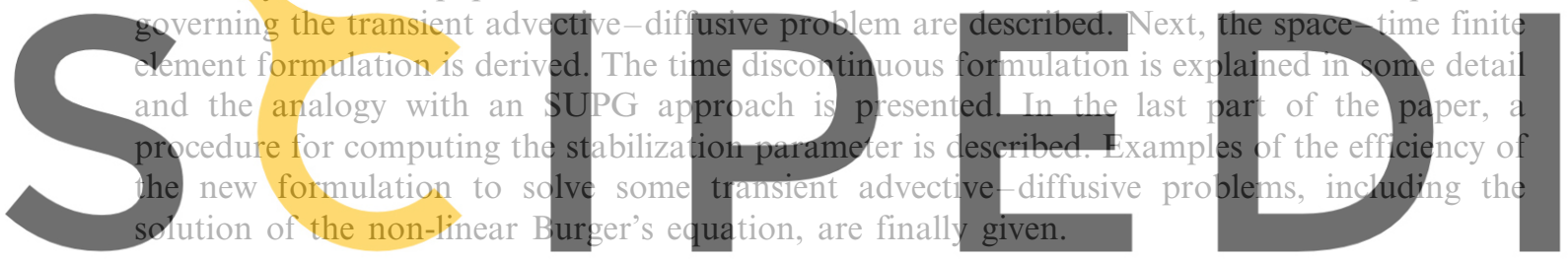
ADVECTIVE-DIFFUSIVE PROBLEMS

Consider the balance of fluxes for the one-dimensional advective-diffusive problem in an arbitrary finite space-tine slab $[x-h, x] \times[t-\delta, t]$, where $h$ is the length of the space balance domain and $\delta$ is a time increment defining the size of the balance domain in the time axis (Figure 1). The global balance law can be written as

$$
\int_{t-\delta}^{t}\left[\int_{x-h}^{x} f \mathrm{~d} x\right] \mathrm{d} t=\int_{x-h}^{x}\left[\int_{t-\delta}^{t} v \mathrm{~d} \phi\right] \mathrm{d} x,
$$

where $f$ denotes the space fluxes, $\phi$ is the transported variable and $v$ is the advective coefficient.

Assuming that both $h$ and $\delta$ are finite and retaining first-order terms in $h$ and $\delta$ only gives (see Appendix A)

$$
\left.r-\frac{h}{2} \frac{\partial r}{\partial x}-\frac{\delta}{2} \frac{\partial r}{\partial t}=0 \quad \text { for } x \in\right] 0, L[, t>0,
$$


with

$$
r=-v\left(\frac{\partial \phi}{\partial t}+u \frac{\partial \phi}{\partial x}\right)+\frac{\partial}{\partial x}\left(\kappa \frac{\partial \phi}{\partial x}\right)+Q,
$$

where $u$ is the advective velocity, $\kappa$ is the diffusive coefficient and $Q$ is a distributed source.

Equation (2) can be considered the stabilized form of the balance differential equation for the transient advective-diffusive problem. Note that for $h=\delta=0$, the standard infinitesimal form of the transient advective-diffusive problem $r=0$ is recovered. Equation (2) is also the basis for deriving numerical schemes ensuring the stability of the solution both in space and time domains. For the sake of preciseness, only the space-time (discontinuous) formulation will be considered here. Note that in all cases the distance $h$ and the time increment $\delta$ in (2) play the role of stabilization parameters, ensuring stability of the numerical solution for the discrete problem. Indeed, the correct evaluation of these parameters is critical and this issue will be discussed in a next section for the space-time formulation.

\section{STABILIZED SPACE-TIME FORMULATION}

\section{Let us transform the time 'direction' $t$ into an auxiliary 'spatial' direction $y^{*}$ by means of a} fictitious 'time velocity' $v^{*}$ so that

$$
y^{*}=v^{*} t
$$

Using this concept, Equation (2) can be rewritten as
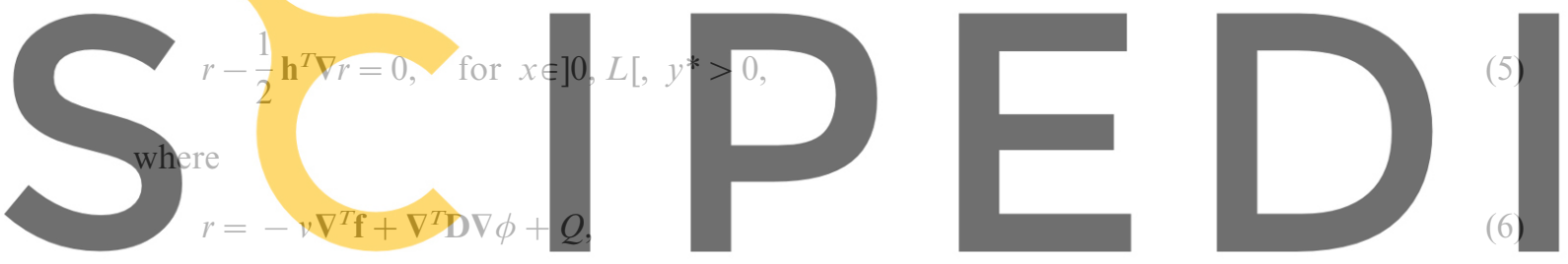

the advective flux vector is Register for free at https//www.scipedia.com to download the version without the

the gradient operator is

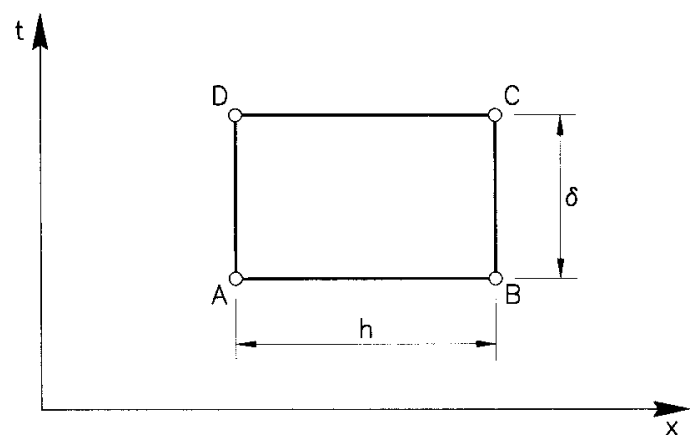

Figure 1. Arbitrary finite space-time slab where balance of fluxes is considered. 


$$
\boldsymbol{\nabla}=\left[\frac{\partial}{\partial x}, \frac{\partial}{\partial y^{*}}\right]^{T}
$$

and the conductivity matrix is

$$
\mathbf{D}=\left[\begin{array}{ll}
\kappa & 0 \\
0 & 0
\end{array}\right] .
$$

The so-called characteristic length vector $\mathbf{h}$ is given by

$$
\mathbf{h}=\left[h, \delta v^{*}\right]^{T} .
$$

The 'intrinsic time' of the space-time problem can be defined now as

$$
\tau=\frac{\bar{h}}{2|\mathbf{u}|},
$$

where the characteristic length $\bar{h}$ is

$$
\bar{h}=\left[h^{2}+\left(\delta v^{*}\right)^{2}\right]^{1 / 2}
$$

and

$$
|\mathbf{u}|=\left[u^{2}+\left(v^{*}\right)^{2}\right]^{1 / 2}, \quad \text { with } \mathbf{u}=\left\{\begin{array}{c}
u \\
v^{*}
\end{array}\right\} .
$$

In the following it will be assumed that the characteristic length vector is aligned with the velocity vector. This implies

$$
\mathbf{h}=\frac{\bar{h}}{|\mathbf{u}|} \mathbf{u}=2 \tau \mathbf{u} \text {. }
$$

(13)

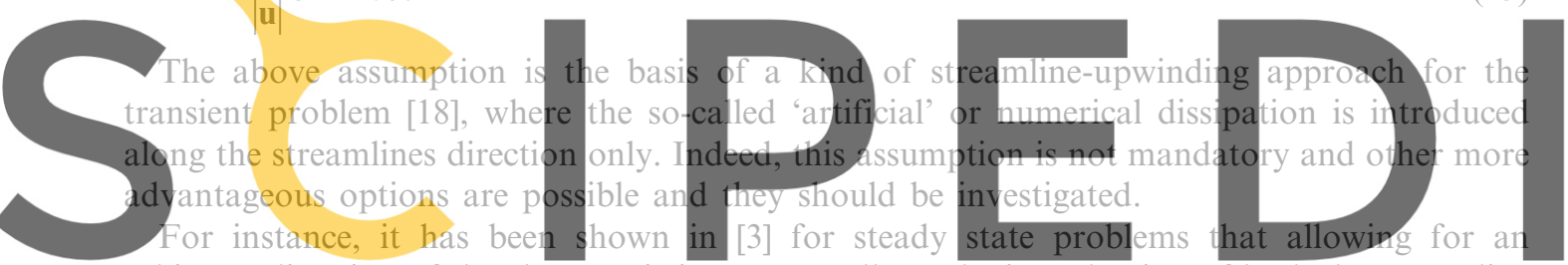

arbitrary direction of the characteristic vector $\mathbf{h}$ allows the introduction of both the streamline

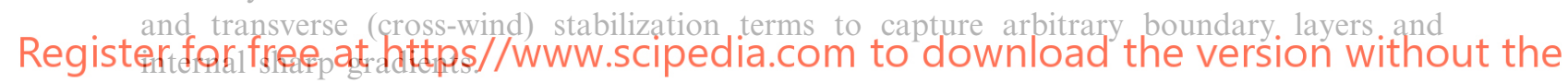

Substituting Equation (11) into (14) gives the alternative form of the stabilized space-time differential equation in terms of the intrinsic time parameter as

$$
r-\tau \mathbf{u}^{T} \nabla r=0
$$

\subsection{Boundary conditions}

Each of the equivalent stabilized forms (2), (5) and (14) has to be solved together with the following boundary conditions

$$
\begin{aligned}
& \phi-\bar{\phi}=0 \quad \text { on } \Gamma_{\phi}, \\
& -v \mathbf{n}^{T} \mathbf{u} \phi+\mathbf{n}^{T} \mathbf{D} \nabla \phi+\bar{q}-\tau \mathbf{u}^{T} \mathbf{n} r=0 \quad \text { on } \Gamma_{q}, \\
& \phi(\mathbf{x}, 0)=\bar{\phi}_{0}, \quad \text { for } t=t_{0} .
\end{aligned}
$$

In the above, $\Gamma_{\phi}$ and $\Gamma_{q}$ are the usual space boundaries, where the variable and the normal flux are prescribed respectively, $\mathbf{n}$ is the normal vector, $\bar{q}$ is the prescribed normal flux at the 


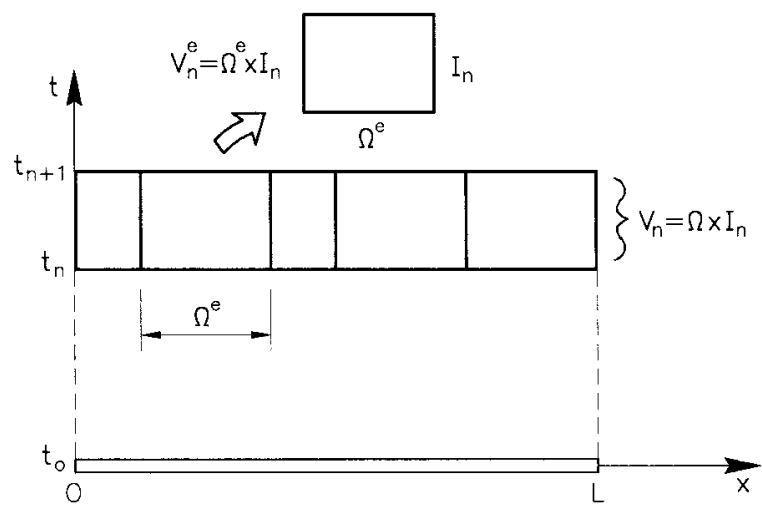

Figure 2. Space-time slab.

Neumann boundary $\Gamma_{q}, \bar{\phi}$ is the prescribed value of the unknown at the Dirichlet boundary $\Gamma_{\phi}$, and $\phi_{0}$ is the known value of $\phi$ at the initial time.

Note that the Neumann boundary condition (16) includes a residual stabilization term. This term appears naturally when considering a higher balance law next to a Neumann boundary. Accounting for this term is critical for consistency of the stabilization process. The derivation of this boundary stabilization term can be found in [1].

\subsection{Finite element approximation}

Consider the partition $0=t_{0}<t_{1} \cdots<t_{n}=T$ of the time interval $\left.I=\right] 0, T[$. Denote the $n$th
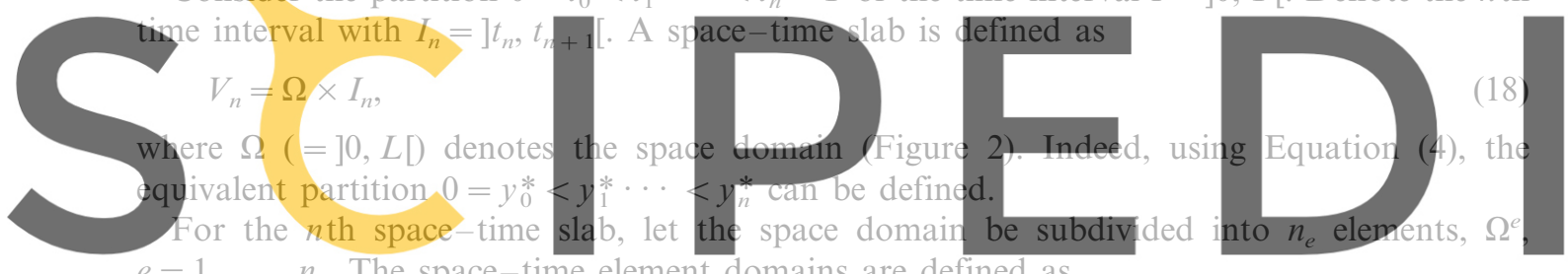

$e=1, \ldots, n_{e}$. The space-time element domains are defined as

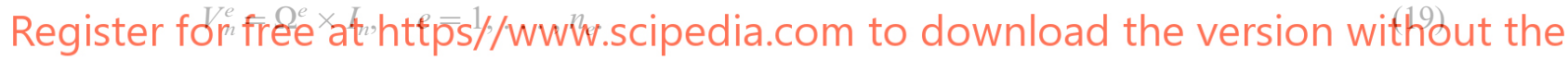

Within each space-time element containing $n$ nodes, the finite element approximation is written as

$$
\phi \simeq \hat{\phi}=\sum_{i=1}^{n} N_{i}\left(x, y^{*}\right) \phi_{i},
$$

where $N_{i}$ are the element shape functions and $\phi_{i}$ are nodal values. The functions $N_{i}$ are assumed $C^{0}$ continuous throughout each space-time slab, but are allowed to be discontinuous across the slab integrals, namely at times $t_{1}, t_{2}, \ldots, t_{N-1}$ (or the equivalent time 'co-ordinates' $y_{1}^{*}, y_{2}^{*}, \ldots, y_{N-1}^{*}$ ). Substituting Equation (20) into Equations (14) and (16) gives

$$
\begin{aligned}
& \hat{r}-\tau \mathbf{u}^{T} \mathbf{\nabla} \hat{r}=r_{V} \quad \text { on } V_{n}, \\
& -v \mathbf{n}^{T} \mathbf{u} \hat{\phi}+\mathbf{n}^{T} \mathbf{D} \boldsymbol{\nabla} \hat{\phi}+\bar{q}-\tau \mathbf{u}^{T} \mathbf{n} \hat{r}=r_{q} \quad \text { on } \Gamma_{q},
\end{aligned}
$$

where $\hat{r}=r(\hat{\phi})$ and $r_{V}$ and $r_{q}$ are the residuals of the approximate solution in the space-time slab $V_{n}$ and the Neumann boundary $\Gamma_{q}$ respectively. As usual in the FEM, the Dirichlet boundary condition (15) will be assumed to be satisfied exactly. 
The weighted residual form of Equations (20) and (21) is

$$
\iint_{V_{n}} w\left(\hat{r}-\tau \mathbf{u}^{T} \nabla \hat{r}\right) \mathrm{d} V+\int_{\Gamma_{q}} \bar{w}\left[-v \mathbf{n}^{T} \mathbf{u} \hat{\phi}+\mathbf{n}^{T} \mathbf{D} \nabla \hat{\phi}+\bar{q}-\tau \mathbf{u}^{T} \mathbf{n} \hat{r}\right] \mathrm{d} \Gamma_{n}=0,
$$

where $w$ and $\bar{w}$ are arbitrary test functions with the same continuity properties as those of the shape functions. As usual, $w=\bar{w}=0$ on $\Gamma_{\phi}$ will be assumed. Integrating by parts the term incorporating $\tau$ in the first integral of Equation (22) and choosing $\bar{w}=-w$ gives

$$
\iint_{V_{n}}\left[w+\nabla^{T}(\tau \mathbf{u} w)\right] \hat{r} \mathrm{~d} V-\int_{\Gamma_{q}} w\left(-v \mathbf{n}^{T} \mathbf{u} \hat{\phi}+\mathbf{n}^{T} \mathbf{D} \nabla \hat{\phi}+\bar{q}\right) \mathrm{d} \Gamma_{n}=0 .
$$

Let us further assume that both the intrinsic time and the velocity are constant within each element (i.e. $\nabla^{T} \tau \mathbf{u}=0$ ). Integrating by parts the diffusive terms in the product $w \hat{r}$ within the first integral of Equation (23) gives

$$
\begin{aligned}
& \iint_{V_{n}}\left[w v \nabla^{T} \mathbf{f}+\left(\nabla^{T} w\right) \mathbf{D} \nabla \hat{\phi}\right] \mathrm{d} V+\iint_{V_{n}} \tau \mathbf{u}^{T} \nabla w\left[v \boldsymbol{\nabla}^{T} \mathbf{f}-\nabla^{T}(\mathbf{D} \nabla \hat{\phi})\right] \mathrm{d} V-\iint_{V_{n}} w Q \mathrm{~d} V \\
& +\int_{\Gamma_{q}} w\left(-v \mathbf{m}^{T} \mathbf{u} \hat{\phi}+\bar{q}\right) \mathrm{d} \Gamma_{n}-\iint_{V_{n}} \tau \mathbf{u}^{T}(\nabla w) Q \mathrm{~d} V=0 .
\end{aligned}
$$

Let us compute the integral along the Neumann boundaries $\Gamma_{q_{n}}$ in Equation (24) for the space-time slab of Figure 3. The total flux is the sum of the advective and diffusive fluxes across the lateral boundaries $\Gamma_{\mathrm{L}}$ and $\Gamma_{\mathrm{R}}$ and the advective flux across the lower boundary $\Gamma_{n}^{+}$ and the upper boundary $\Gamma_{n+1}^{-}$.

The normal flux $\bar{q}$ on the lower and upper boundaries $\Gamma_{n}^{+}$and $\Gamma_{n+1}^{-}$can be computed from the advective velocity $v^{*}$ as
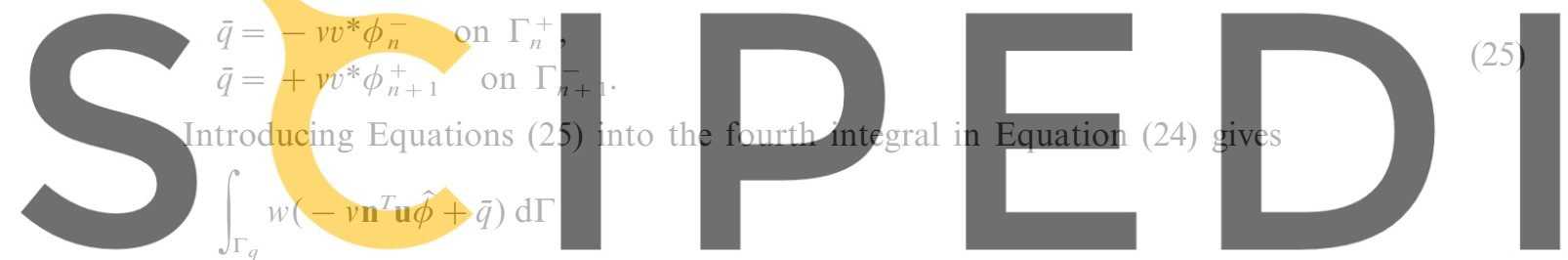

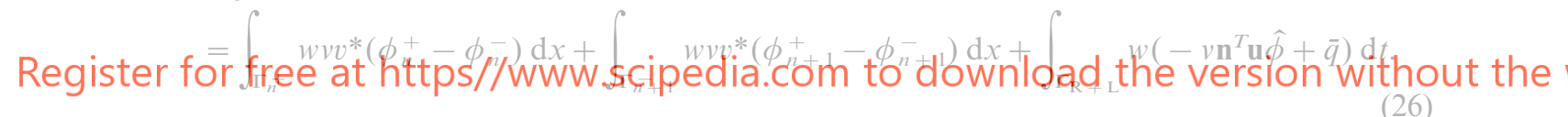

The first integral of the right-hand-side of Equation (26) gives the so-called jump conditions in a discontinuous approximation in time of the unknown $\phi$. The second integral can be set equal

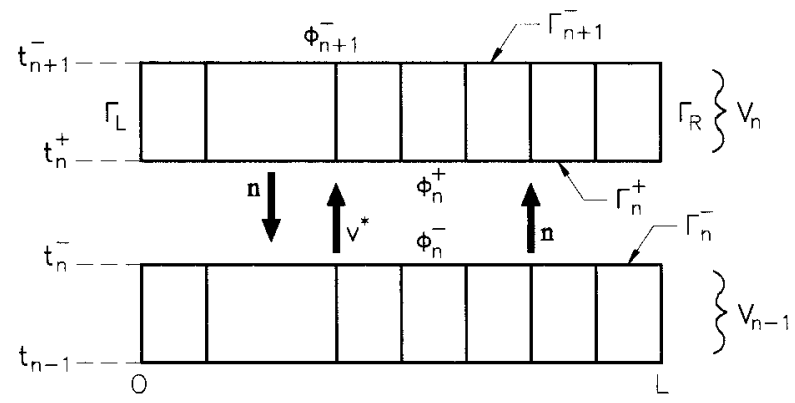

Figure 3. Space-time slab. Definition of Neumann boundaries where the flux is prescribed. 

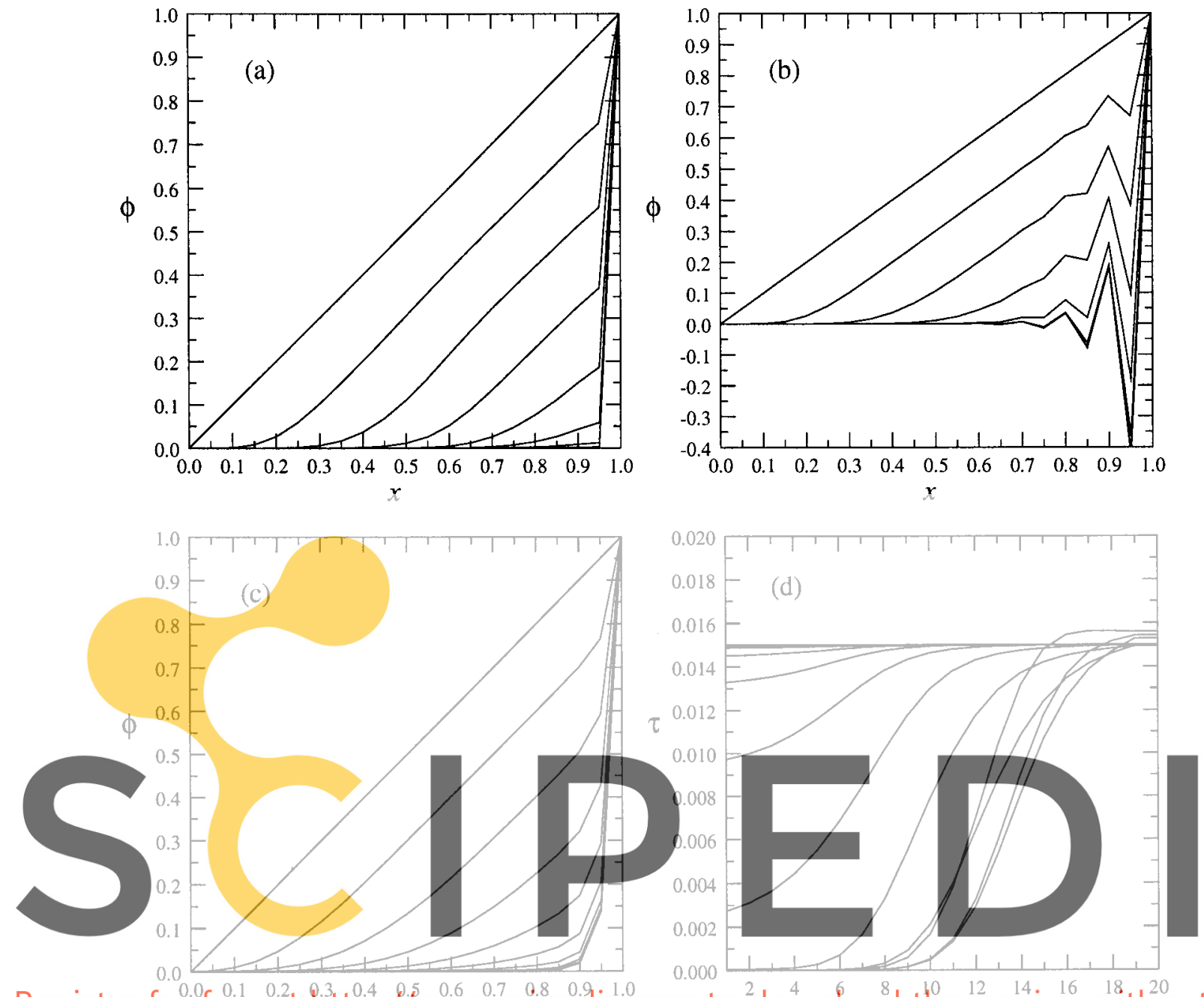

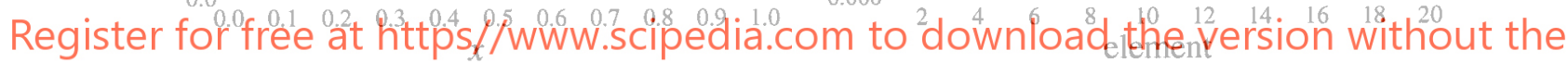

Figure 4. Example 1: solution of the advection-diffusion problem with $C_{0}=1$, using (a) automatic computation of $\tau$, (b) Galerkin method, (c) $\tau$ as defined by Equation (35), (d) time evolution of the distribution of $\tau$ as obtained with present method.

to zero by assuming $\phi_{n+1}^{-}=\phi_{n+1}^{+}$, while solving the equations for the time slab. Using this latter assumption and substituting Equation (26) into (24) gives the final expression for the stabilized integral form as

$$
\begin{aligned}
& \iint_{V_{n}}\left[w v \nabla^{T} \mathbf{f}+\left(\nabla^{T} w\right) \mathbf{D} \nabla \hat{\phi}\right] \mathrm{d} V+\iint_{V_{n}} \tau \mathbf{u}^{T} \nabla w\left[v \nabla^{T} \mathbf{f}-\nabla^{T}(\mathbf{D} \nabla \hat{\phi})\right] \mathrm{d} V \\
& +\int_{\Gamma_{n}^{+}} w v v^{*}\left(\phi_{n}^{+}-\phi_{n}^{-}\right) \mathrm{d} x-\iint_{V_{n}} w Q \mathrm{~d} V+\int_{\Gamma_{\mathrm{R}+\mathrm{L}}} w\left(-v \mathbf{n}^{T} \mathbf{u} \hat{\phi}+\bar{q}\right) \mathrm{d} t \\
& -\iint_{V_{n}} \tau \mathbf{u}^{T}(\nabla w) Q \mathrm{~d} V=0 .
\end{aligned}
$$


For the sake of clarity let us rewrite the expanded form of Equation (27) taking $v^{*}=1$, i.e. $y^{*}=t$. This gives

$$
\begin{aligned}
& \iint_{V_{n}}\left(v w \frac{\partial \hat{\phi}}{\partial t}+v w \frac{\partial \hat{\phi}}{\partial x}+\frac{\partial w}{\partial x} \kappa \frac{\partial \hat{\phi}}{\partial x}\right) \mathrm{d} V+\iint_{V_{n}}\left(v \frac{\partial w}{\partial t}+u \frac{\partial w}{\partial x}\right) \tau \hat{r} \mathrm{~d} V \\
& +\int_{\Gamma_{n}^{+}} w v v^{*}\left(\phi_{n}^{+}-\phi_{n}^{-}\right) \mathrm{d} x-\iint_{V_{n}} w Q \mathrm{~d} V+\int_{\Gamma_{\mathrm{R}+\mathrm{L}}} w\left(-v \mathbf{n}^{T} \mathbf{u} \hat{\phi}+\bar{q}\right) \mathrm{d} t \\
& -\iint_{V_{n}} \tau\left(v \frac{\partial w}{\partial t}+u \frac{\partial w}{\partial x}\right) Q \mathrm{~d} V=0 .
\end{aligned}
$$

The first, fourth and fifth integrals in Equations (27) and (28) constitute the standard Galerkin formulation. The second and last integrals constitute the stabilization terms arising in a natural form from the original stabilized differential equations. Note the equivalence of these terms with an analogous SUPG space-time formulation [18]. The third integral is the standard jump condition derived from the lack of continuity of the unknown variable across the upper and lower slab interfaces. The jump condition imposes a weakly enforced continuity across these interfaces and is the mechanism by which information is propagated from one space-time slab to another. Equation (27) (or (28)) leads to a discretized system of equations, where the unknowns are the nodal values of $\phi_{n}^{+}$and $\phi_{n+1}^{-}$at the boundaries $\Gamma_{n}^{+}$and $\Gamma_{n+1}^{-}$respectively (see Figure 3).

\section{Remark 1}

The choice of a continuous approximation in time leads to the same stabilized expressions given by Equations (27) and (28), where the third integral imposing slab continuity now vanishes.
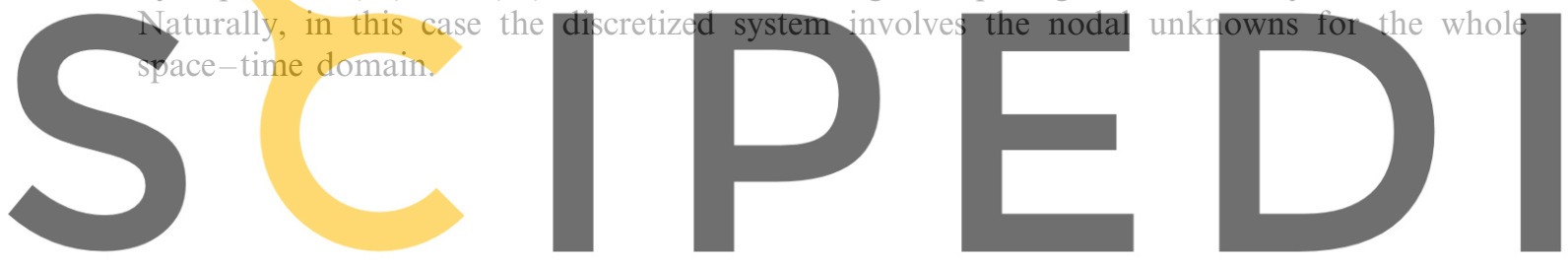

Register for free at https//www.scipedracom to download the version without the

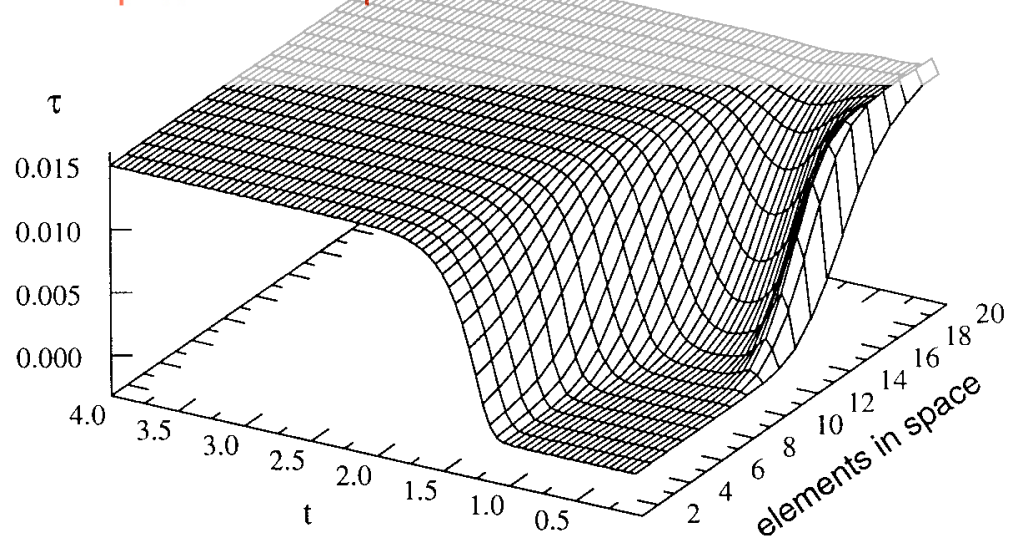

Figure 5. Solution of the advection-diffusion problem with $C_{0}=1$. Time evolution of the stabilization parameter $\tau$ for each element. 

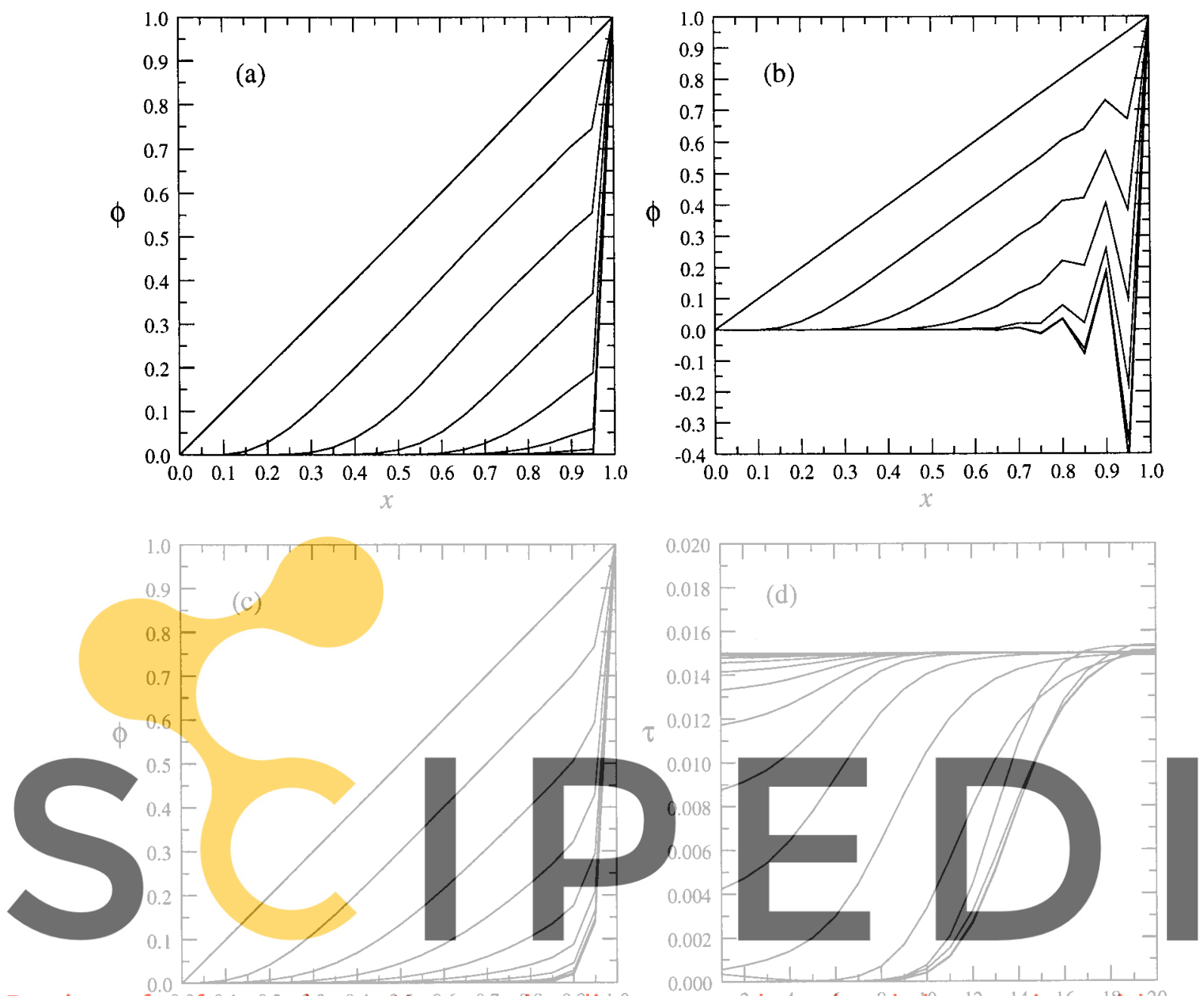

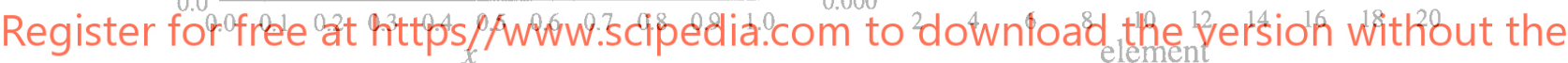

Figure 6. Example 1: solution of the advection-diffusion problem with $C_{0}=2$, using (a) automatic computation of $\tau$, (b) Galerkin method, (c) $\tau$ as defined by Equation (35), (d) time evolution of the distribution of $\tau$ as obtained with the present method.

\section{COMPUTATION OF THE STABILIZATION PARAMETER}

The method to compute the stabilization parameter is an extension of the approach proposed in [2,3] for steady state advective-diffusion problems. The basic steps are summarized below.

Let us define the average residual for a particular numerical solution over a space-time element as

$$
r^{(e)}=\frac{1}{V_{h}^{e}} \iint_{V_{n}^{e}} r_{V} \mathrm{~d} V
$$

Substituting Equation (21) into (29) gives 


$$
r^{(e)}=\hat{r}^{(e)}-\tau^{(e)}\left(\mathbf{u}^{T} \nabla \hat{r}\right)^{(e)} .
$$

Note that in Equation (30) it has been assumed that the intrinsic time parameter is constant within each space-time element. Obviously, Equation (30) can be further simplified if the velocity $\mathbf{u}$ is also taken as constant within each element.

Let us consider that an enhanced solution has been found for a given finite element mesh. This can be achieved by projecting into the original space-time mesh an improved solution obtained by global/local smoothing or superconvergent recovery of derivatives [20,21]. If $r_{1}^{(e)}$ and $r_{2}^{(e)}$ denote the space-time element residuals of the original and the enhanced numerical solution for a given mesh, than it is obvious that

$$
r_{1}^{(e)}-r_{2}^{(e)} \geq 0 \text {. }
$$

Equation (31) holds for $r_{1}^{(\mathrm{e})}>0$. Obviously for $r_{1}^{(\mathrm{e})}<0$ the inequality sign should be reversed. Combining Equations (30) and (31) yields an expression for the intrinsic time of a space-time element as

$$
\tau^{(e)} \geq\left(r_{2}^{(e)}-r_{1}^{(e)}\right)\left[\left(\mathbf{u}^{T} \nabla \hat{r}_{2}\right)^{(e)}-\left(\mathbf{u}^{T} \mathbf{\nabla} \hat{r}_{1}\right)^{(e)}\right]^{-1} .
$$

The equality case in Equation (32) gives the critical value of the element intrinsic time ensuring no growth of numerical error [1].

The following iterative scheme can now be implemented to compute the intrinsic time parameter in order to obtain a stable numerical solution both in space and time.

Step 1. Solve the stabilized problem defined by Equations (27) or (28) to find $\phi_{n}^{+}$and $\phi_{n+1}^{-}$ with an initial guess of $\tau^{(e)}={ }^{0} \tau_{n}^{(e)}$ In the examples shown next ${ }^{0} \tau_{n}^{(e)}={ }^{0} \tau_{n-1}^{(e)}$ (with ${ }^{0} \tau_{1}^{(e)}=0$ ) has been chosen. Compute $r_{1}^{(e)}$ and $\nabla r_{1}^{(e)}$.

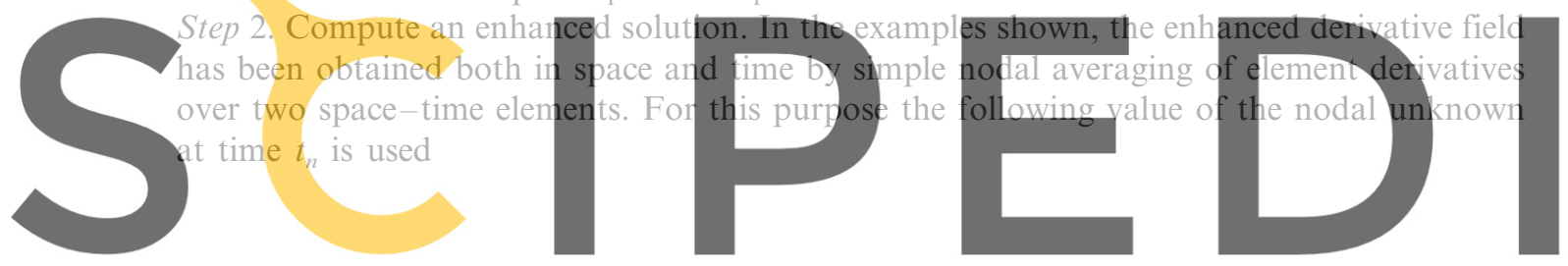

Register for free at https//www.scion the version without the

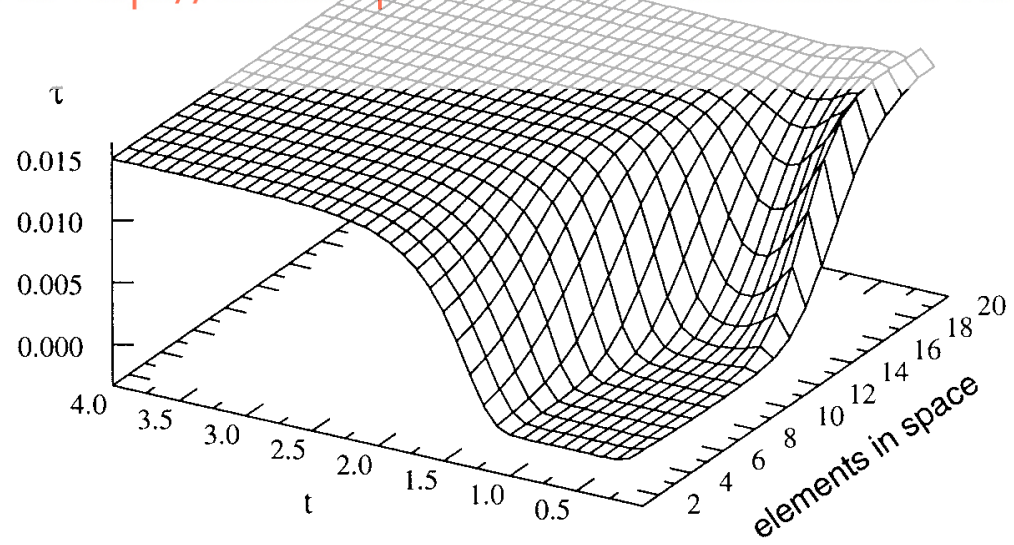

Figure 7. Example 1: solution of the advection-diffusion problem with $C_{0}=2$. Time evolution of the stabilization parameter for each element. 

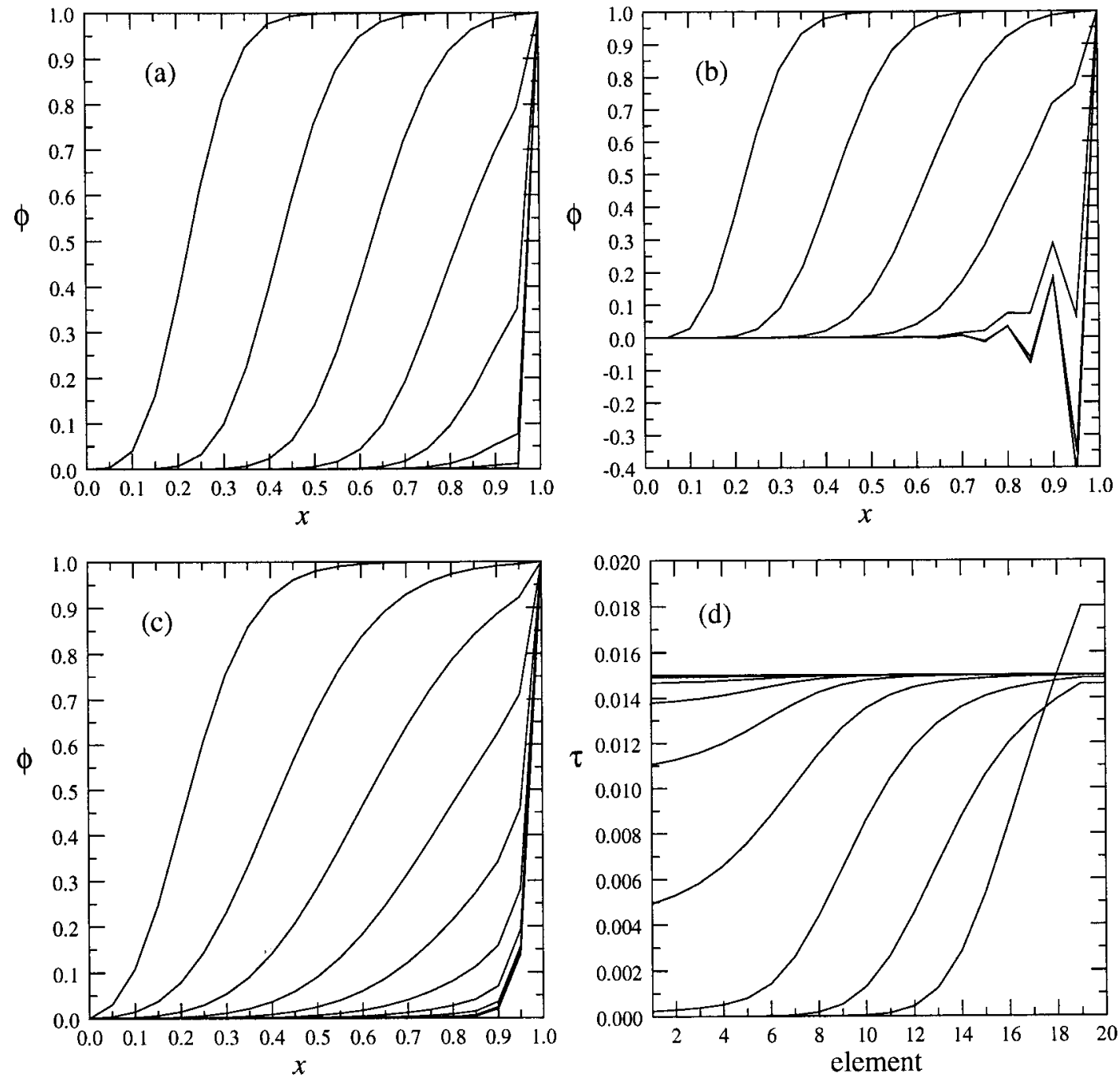

Figure 8. Example 2: solution of the advection-diffusion problem with $C_{0}=1$, using (a) automatic computation of $\tau$, (b) Galerkin method, (c) $\tau$ as defined by Equation (35), (d) time evolution of the distribution of $\tau$ as obtained with the present method.

$$
\phi_{n}=\frac{1}{2}\left(\phi_{n}^{-}+\phi_{n}^{+}\right) .
$$

Step 3. Compute $r_{2}^{(e)}$ and $\nabla r_{2}^{(e)}$.

Step 4. Compute an enhanced value of the element intrinsic time ${ }^{1} \tau_{n}$ using Equation (32). Step 5. Compute a new value of $\tau^{(e)}$ by $\tau^{(e)}=\left({ }^{1} \tau_{n}^{(e)}-{ }^{0} \tau_{n}^{(e)}\right) \beta+{ }^{0} \tau^{(e)}$, where $\beta$ is a relaxation parameter. For the examples that were solved $\beta=0.8$ has been taken.

Step 6. Repeat steps $1-5$ using the updated intrinsic time values until convergence for $\tau^{(e)}$ is found or else a satisfactory numerical solution is obtained.

Steps 1-6 are repeated for each time step. In the examples solved it has been found useful to smooth the distribution of the ${ }^{i} \tau^{(e)}$ values obtained after Step 5. This has been simply done 
again by nodal averaging. It is also noted that above iterative process converges quite fast for well-developed transient solutions (error in the quadratic norm for $\tau^{(e)}$ is less than $1 \%$ obtained in two or three iterations) as the initial guess for $\tau^{(e)}$ is taken as the converged value from previous time step. The convergence in the first time step can be accelerated by using for ${ }^{0} \tau^{(e)}$ the critical value from the simple steady state sourceless advective-diffusive case solved with linear elements (i.e.

$$
\tau^{(e)}=\frac{l^{(e)}}{2 u}\left(1-\frac{1}{\gamma^{(e)}}\right)
$$

with the Peclet number $\left.\gamma^{(e)}=u l^{(e)} / 2 \kappa\right)$.

\section{Remark II}

The above iterative scheme can be interpreted as a particular class of adaptive methods, whereby the numerical solution within each time step is progressively enhanced by means of the computation of improved values of the stabilization parameter, while keeping the mesh and the space-time finite element approximation unchanged.

\section{EXAMPLES}

\subsection{Example 1}

In this example, the advection-diffusion problem

$$
\frac{\partial \phi}{\partial x}+u \frac{\partial \phi}{\partial x}-k \frac{\partial^{2} \phi}{\partial x^{2}}=0
$$

with the initial and boundary conditions

$$
\begin{aligned}
& \phi\left(x, t_{0}\right)=x, \\
& \phi(0, t)=0, \\
& \phi(L, t)=1,
\end{aligned}
$$

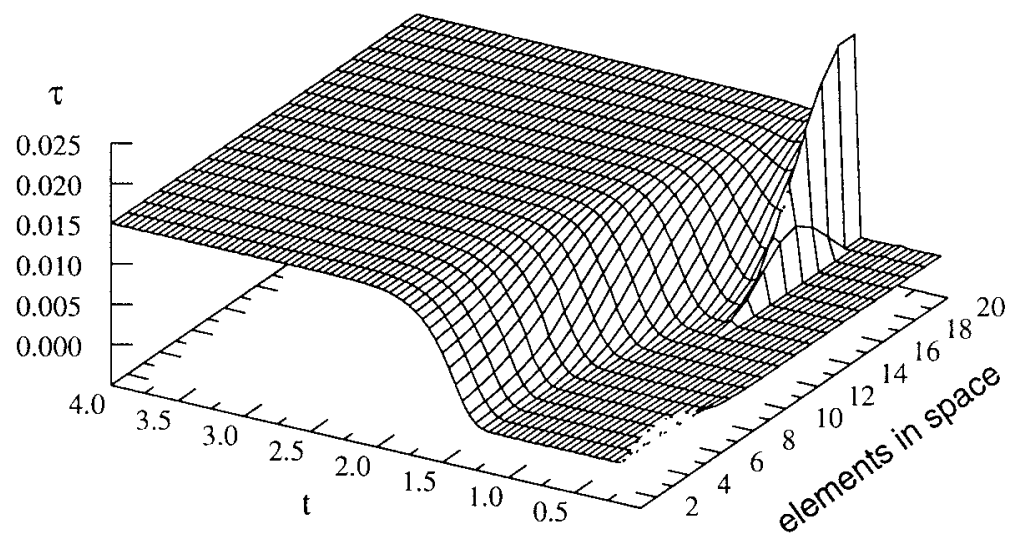

Figure 9. Example 2: solution of the advection-diffusion problem with $C_{0}=1$. Time evolution of the stabilization parameter for each element. 

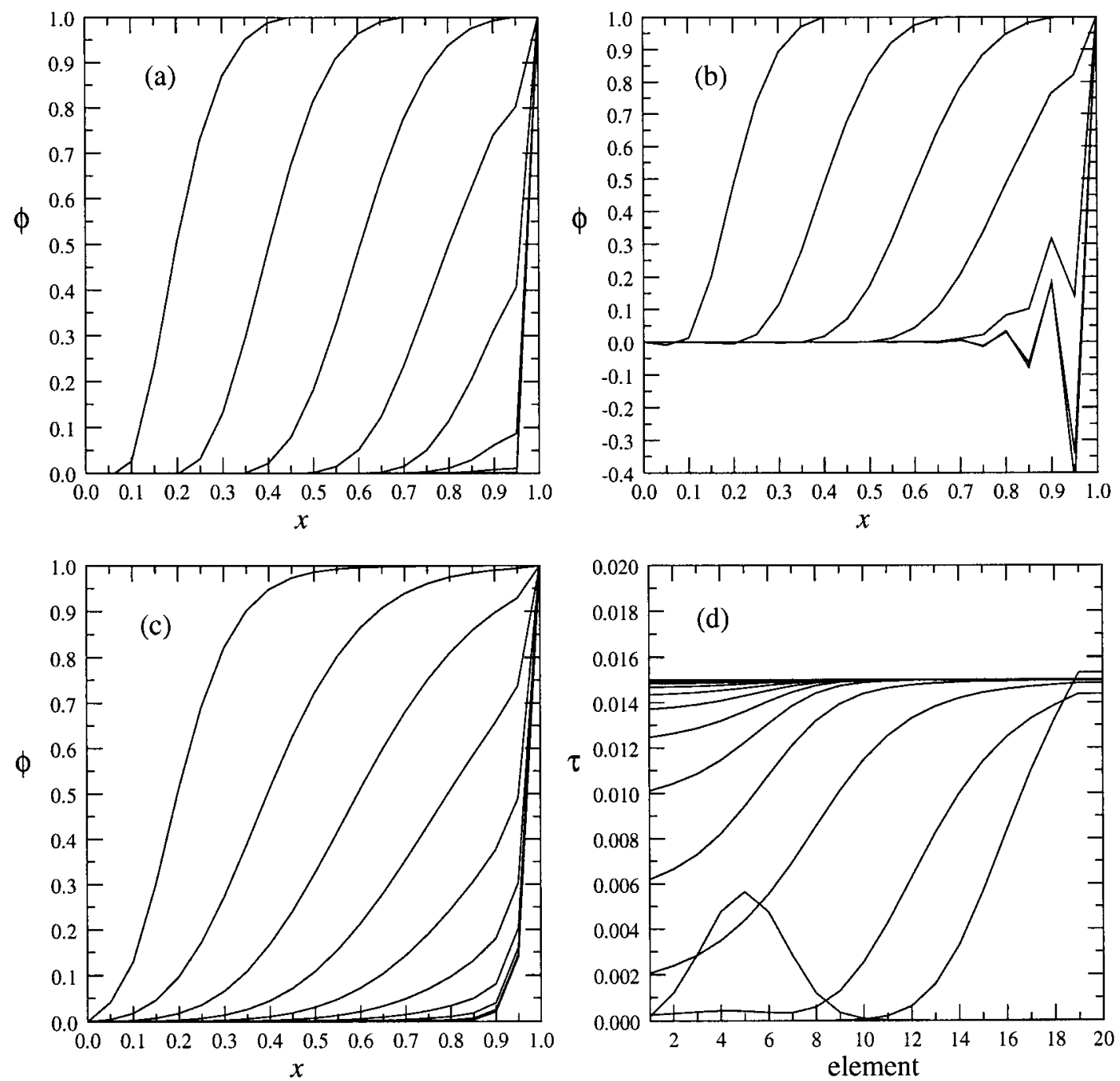

Figure 10. Example 2: Solution of the advection diffusion problem with $C_{0}=2$, using (a) automatic computation of $\tau$, (b) Galerkin method, (c) $\tau$ as defined by Equation (35), (d) time evolution of the distribution of $\tau$ as obtained with the present method.

is presented. The data of the problem are $L=1, u=1, k=0.01$. The discretization in the space-time domain has been carried out using 24 node bilinear square elements with dimensions in space and time equal to 0.05 . Two time steps have been used for the simulations: $\Delta t=0.05$ and $\Delta t=0.1$. Using Equation (4), the 'time velocities' are respectively $v^{*}=1$ and $v^{*}=0.5$. The resulting element Peclet number, $\gamma$, is 2.5 and the Courant numbers for the two cases considered are respectively $C_{0}=1$ and $C_{0}=2$.

The numerical results are presented in Figures 4-7. In Figures 4 and 5 the value of $\phi$ is plotted at times from 0 to 4 in steps of 0.5 obtained with (a) the automatic computation of the stabilization parameter $\tau$ using the iterative adaptive scheme described in previous sections, (b) using the standard non-stabilized Galerkin method [16], and (c) using the expression for $\tau$ given by Shakib [22] 


$$
\tau=\left[\left(\frac{2 u}{\Delta x}\right)^{2}+\left(\frac{4 k}{\Delta x^{2}}\right)^{2}\right]^{-1 / 2},
$$

where $\Delta x$ is the spatial element length. The time evolution of $\tau$ is represented in Figures $4(\mathrm{~d})$ and $5\left(C_{0}=1\right)$ and $6(\mathrm{~d})$ and $7\left(C_{0}=2\right)$.

As expected, the Galerkin method lacks stability in both cases, producing spurious oscillations at all time steps, including the stationary state. On the other hand, the solution utilizing the stabilization parameter given by Equation (35) shows higher diffusivity, failing to reach the correct solution at steady state. The proposed method stabilizes the solution and seems to give the correct distribution of $\phi$ at all times.

In Figures 5 and 7 the distribution of $\tau$ has been plotted against the time and the element number for the two Courant number values. Note that the distribution is not constant over the space domain, especially at the early stages of the analysis. The optimal steady state value for the SUPG formulation $(\tau=0.015)$ is reached in both cases.

\subsection{Example 2}

Here the same advection-diffusion problem of Example 1 is solved but with the initial condition

$$
\phi\left(x, t_{0}\right)=1 .
$$

The parameters are the same as in Example 1 and again the two time steps $\Delta t=0.05$ and $\Delta t=0.1$ are considered. The results are presented in Figures $8-10$. In Figures 8 and 10 the values of $\phi$ and $\tau$ obtained with the iterative adaptive method described in this paper are plotted. The solution develops smoothly during the initial time steps, thus for a while even the Galerkin solution does not show any spurious oscillation. Unsatisfactory results are evident only when the discontinuity reaches the right boundary. In contrast, the results obtained using the stabilization parameter $\tau$ as defined by Equation (35) prove to be over diffusive. The solution obtained with the automatic parameter computation proposed here shows a smooth and less diffusive values at all time steps reaching the correct distribution at steady state.

The space-time distributions of $\tau$ are presented in Figures 9 and 11. It is interesting to see how the new strategy proposed here is able to detect the presence of spurious oscillations and to correct the solution only when needed, computing the correct value of the stabilization

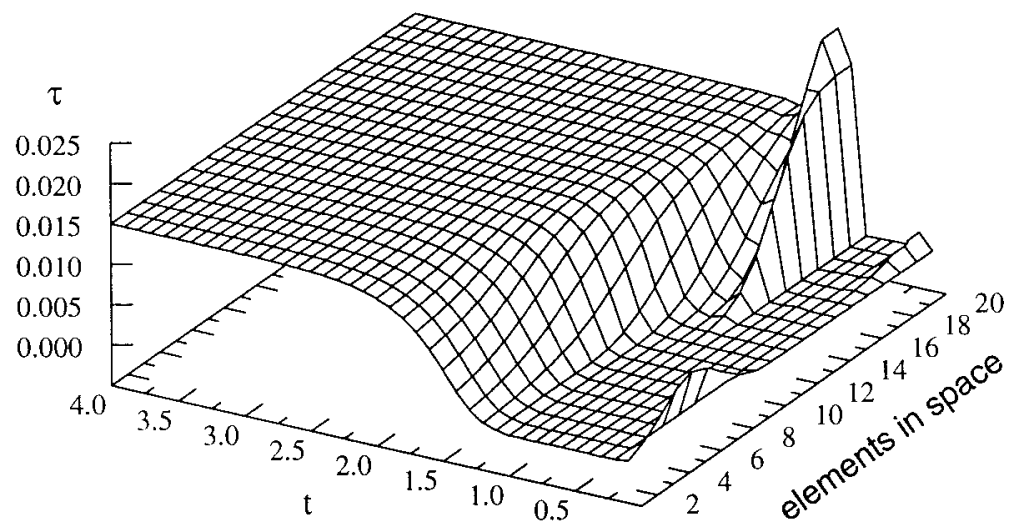

Figure 11. Example 2: solution of the advection-diffusion problem with $C_{0}=2$. Time evolution of the stabilization parameter for each element. 

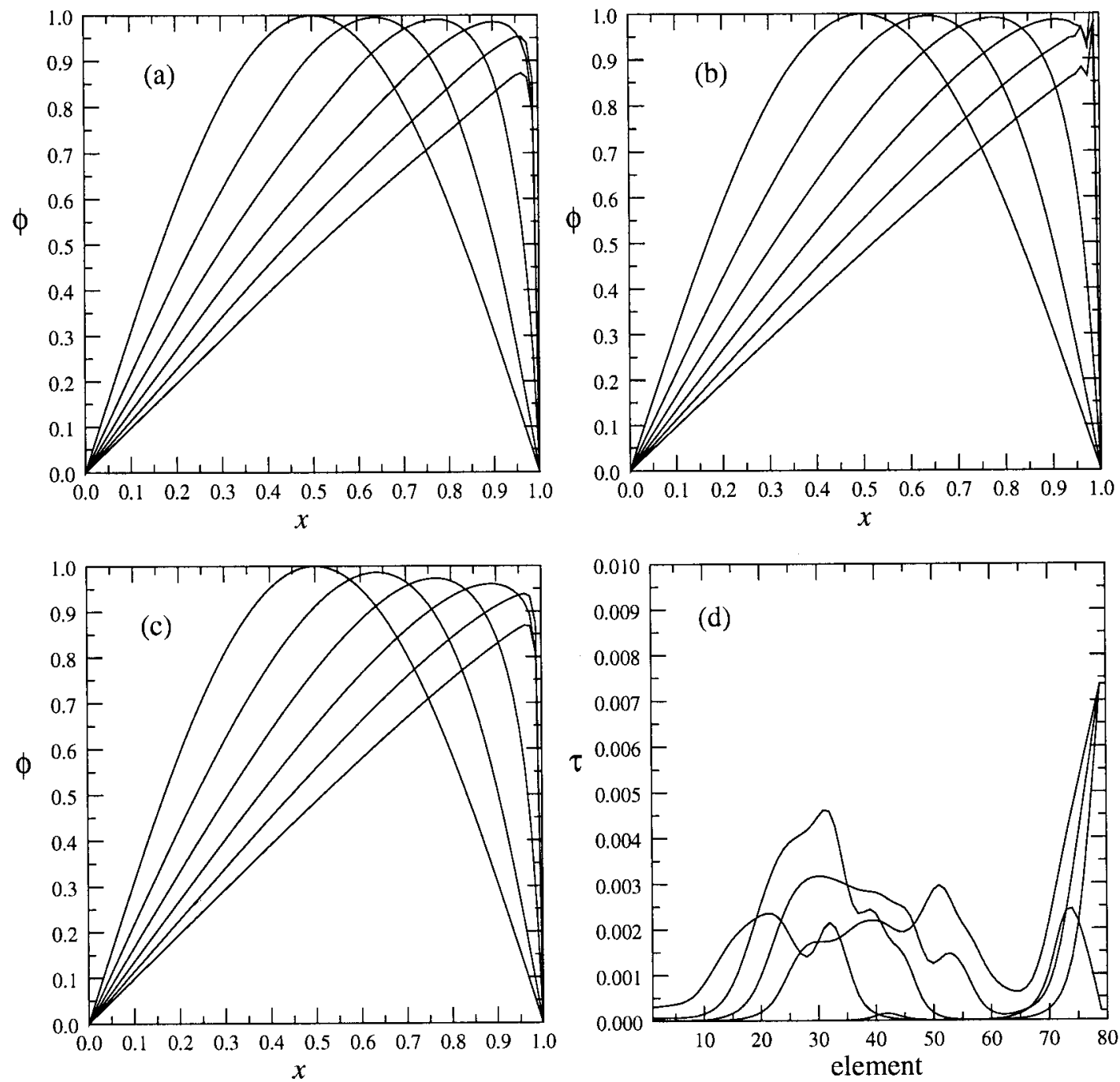

Figure 12. Example 3: solution of the Burger equation, using (a) automatic computation of $\tau$, (b) Galerkin method, (c) $\tau$ as defined by Equation (35), (d) time evolution of the distribution of $\tau$ as obtained with the present method.

parameter $\tau$. Again the optimal steady state value $\tau=0.015$ for the SUPG method is found in both cases.

\subsection{Example 3}

In this example, the method derived for the advection-diffusion equation is applied to the non-linear Burger equation

$$
\frac{\partial \phi}{\partial x}+\phi \frac{\partial \phi}{\partial x}-k \frac{\partial^{2} \phi}{\partial x^{2}}=0
$$

with the initial and boundary conditions 


$$
\begin{aligned}
& \phi\left(x, t_{0}\right)=\sin \pi x, \\
& \phi(0, t)=0, \\
& \phi(L, t)=0,
\end{aligned}
$$

with $k=\frac{1}{100} \pi$. The discretization of the space time domain has been carried out using 80 equally spaced four-nodal rectangular elements. The dimension in space is $\Delta x=0.0125$ and the dimension in time is $\Delta y=1$. The time step has been taken as $\Delta t=4.67 \times 10^{-3}$. The 'space velocity' is, therefore, $v^{*}=214.13$.

The numerical solution is shown in Figure 10 at times from 0 to 0.7 for $\Delta t=0.14$, using the different methods described in Example 1. As with the previous examples, the solution develops smoothly during the initial time steps, thus even the standard Galerkin solution does not show any spurious oscillation. Again, unsatisfactory results are evident only when the maximum value reaches the right boundary (Figure 12(b)).

The results obtained using the parameter $\tau$ as defined by Equation (35) prove again to be over diffusive, especially when the solution is smooth (Figure 12(c)). The solution obtained with the automatic parameter computation proposed in the paper are smooth and less diffusive for all time steps.

The space-time distribution of $\tau$ depicted in Figure 13 reveals that the stabilization parameter has a significant value only for the elements that show a lack of stability in the Galerkin solution.

\section{CONCLUDING REMARKS}

The new stabilized form of the transient governing differential equations derived via the 'finite increment calculus' approach proposed in [1] seems to be a natural starting point for formulating stable finite element methods for transient advective-diffusive problems. The method has been applied in this paper to the space-time formulation using a time discontinuous approximation. Applications to derive other class of stabilized FEM time integration schemes are also possible and will be investigated. The new stabilized governing equations are the basis for computing the stabilization parameter in an iterative manner. This approach can

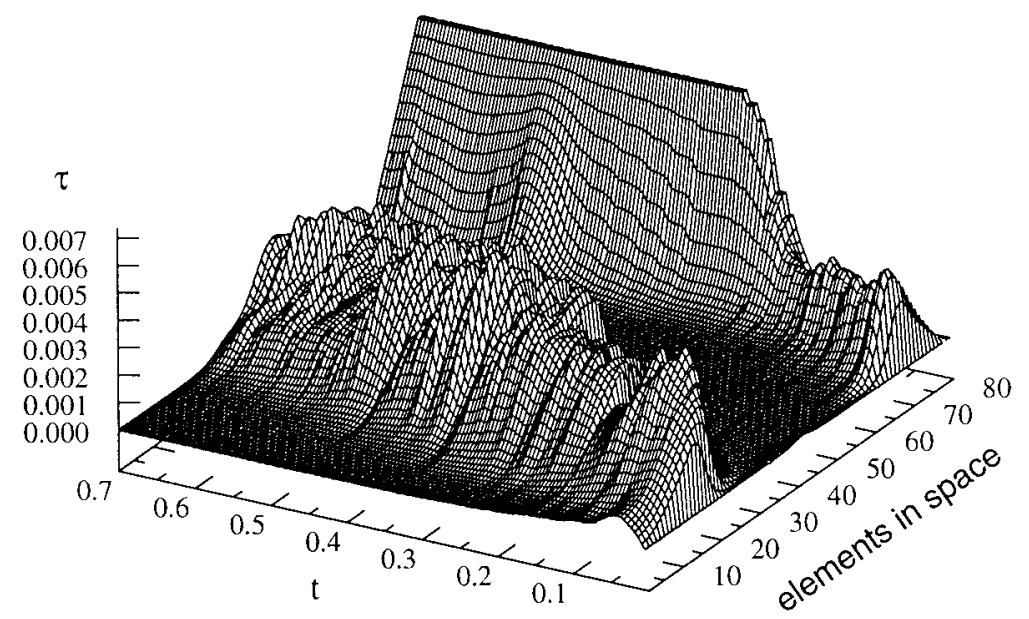

Figure 13. Example 3: solution of the Burger equation. Time evolution of the stabilization parameter for each element. 
be interpreted as a class of adaptive method where the numerical solution is enhanced by progressively improving the value of the stabilization parameter, while keeping the mesh and the finite element approximation unchanged. The efficiency of this stabilization procedure has been shown for obtaining accurate transient and steady state solutions for linear and non-linear (Burger equation) advective-diffusive problems using simple bilinear space-time (discontinuous) elements.

\section{APPENDIX A}

\section{A.1. Derivation of stabilized differential equations via a finite calculus approach}

Let us write the balance equation for the one-dimensional advection-diffusion equation for an arbitrary space-time slab of finite dimensions $[x-h, x] \times[t-\delta, t]$, where $h$ and $\delta$ are the dimensions of the domain in space and time axes respectively (Figure 1). The balance of fluxes in the space-time domain can be written as

$$
\int_{t-\delta}^{t} f \mathrm{~d} t=\int_{x-h}^{x}\left[\int_{t-\delta}^{t} v \delta \phi\right] \mathrm{d} x,
$$

where $f$ is the sum of fluxes in the space direction. The value of $f$ can be obtained as follows:

$$
f=q_{B}-q_{A}+\int_{x-h}^{x} Q \mathrm{~d} x,
$$

with

$$
q_{i}=q_{\mathrm{a}_{i}}+q_{\mathrm{d}_{i}}
$$

where $q_{\mathrm{a}}$ and $q_{\mathrm{d}}$ represent the advective and diffusive fluxes given by

$$
q_{\mathrm{a}}=v u \phi \quad \text { and } \quad q_{\mathrm{d}}=-\kappa \frac{\mathrm{d} \phi}{\mathrm{d} x} .
$$

The fluxes $q_{A}$ are expressed now in terms of the fluxes $q_{B}$ using the following Taylor's expansion

$$
q_{A}=q_{B}-\left.h \frac{\partial q}{\partial x}\right|_{B}+\left.\frac{h^{2}}{2} \frac{\partial^{2} q}{\partial x}\right|_{B} .
$$

Substituting Equation (A.5) into (A.2) and making use of Equations (A.3) and (A.4) and assuming a linear distribution of the source $Q$ within the space domain gives, after little algebra,

$$
f=\left[r_{1}-\frac{h}{2} \frac{\mathrm{d} r_{1}}{\mathrm{~d} x}\right] h,
$$

with

$$
r_{1}=-v u \phi+\frac{\partial}{\partial x}\left(\kappa \frac{\partial \phi}{\partial x}\right)+Q .
$$

Note that index $B$ has been suppressed from the right-hand-side terms in Equation (A.6) as the position of point $B$ is assumed to be arbitrary.

The left-hand-side of Equation (A.1) can now be approximated using Equation (A.7) as 


$$
\begin{aligned}
\int_{t-\delta}^{t} f \mathrm{~d} t & =\int_{t-\delta}^{\delta}\left[r_{1}-\frac{h}{2} \frac{\partial r_{1}}{\partial x}\right] h \mathrm{~d} t=\frac{h \delta}{2}\left[\left(r_{1}-\frac{h}{2} \frac{\partial r_{1}}{\partial x}\right)^{t}+\left(r_{1}-\frac{h}{2} \frac{\partial r_{1}}{\partial x}\right)^{t-\delta}\right] \\
& =h \delta\left[\left(r_{1}-\frac{h}{2} \frac{\partial r_{1}}{\partial x}\right)^{t}-\frac{\delta}{2} \frac{\partial}{\partial t}\left(r_{1}-\frac{h}{2} \frac{\partial r_{1}}{\partial x}\right)^{t}\right] .
\end{aligned}
$$

In the derivation of Equation (A.8), $f$ has been assumed to vary linearly in the time interval. Also, use of a second-order Taylor's expansions in time of the term evaluated at $t-\delta$ has been made.

The right-hand-side of Equation (A.1) can similarly be expressed (assuming $v$ to be constant) as

$$
\begin{aligned}
\int_{x-h}^{x}\left[\int_{t-\delta}^{t} v \mathrm{~d} \phi\right] \mathrm{d} x & =\int_{x-h}^{h} v\left[\phi^{t}-\phi^{t-\delta}\right] \mathrm{d} x=\int_{x-h}^{h} v\left[\delta \frac{\partial \phi}{\partial t}-\frac{\delta^{2}}{2} \frac{\partial^{2} \phi}{\partial t^{2}}\right] \mathrm{d} x \\
& =v \delta h\left[\left(\frac{\partial \phi}{\partial t}-\frac{\delta}{2} \frac{\partial^{2} \phi}{\partial t^{2}}\right)^{t}-\frac{h}{2} \frac{\partial}{\partial x}\left(\frac{\partial \phi}{\partial t}-\frac{\delta}{2} \frac{\partial^{2} \phi}{\partial t^{2}}\right)\right] .
\end{aligned}
$$

Noting that the time $t$ is also arbitrary and equalling Equations (A.8) and (A.9) gives, after simplification, the final form of the stabilized transient balance equation as

$$
r-\frac{h}{2} \frac{\partial r}{\partial x}-\frac{\delta}{2} \frac{\partial}{\partial t}\left(r-\frac{h}{2} \frac{\partial r}{\partial x}\right)=0,
$$

with

$$
r=-v \frac{\partial \phi}{\partial t}+r_{1}
$$

Retaining first-order terms in $h$ and $\delta$ in Equation (A.10) gives the stabilized Equation (2) used in the paper.

Note that by assuming the dimensions of the balance space-time slab to be infinitesimal, the standard governing equation for the transient advective-diffusive problem is recovered as

$$
r=0,
$$

or

$$
v \frac{\partial \phi}{\partial t}+v u \frac{\partial \phi}{\partial x}-\frac{\partial}{\partial x}\left(\kappa \frac{\partial \phi}{\partial x}\right)-Q=0 .
$$

Further details on the finite increment calculus approach used here can be found in [1].

\section{REFERENCES}

1. E. Oñate, 'Derivation of stabilized equations for advective-diffusive transport and fluid flow problems', Comput. Methods Appl. Mech. Eng., 151, $233-267$ (1998).

2. E. Oñate, J. García and S. Idelsohn, 'Computation of the stabilization parameter for the finite element solution of advective-diffusive problems', Int. J. Numer. Methods Fluids, 25, 1385-1407 (1997).

3. E. Oñate, J. García and S. Idelsohn, 'An alpha-adaptive approach for stabilized finite element solution of advective-diffusive problems with sharp gradients', in P. Ladeveze and J.T. Oden (eds.), New Advances in Adaptive Computational Methods in Mechanics, Elsevier, Amsterdem, 1998.

4. C. Johnson, U. Nävert and J. Pitkärantas, 'Finite element methods for linear hyperbolic systems', Comp. Methods Appl. Mech. Eng., 45, 285-312 (1984).

5. C. Johnson and J. Saranen, 'Streamline diffusion methods for the incompressible Euler and Navier-Stokes equations', Math. Comput., 47, 1-8 (1986).

6. T.E. Tezduyar and D.K. Ganjoo, 'Petrov-Galerkin formulations with weighting functions dependent upon spatial and temporal discretization: applications to transient convection-diffusion problems', Comp. Methods Appl. Mech. Eng., 59, 49-71 (1986). 
7. C. Johnson, A. Srepessy and P. Hanslov, 'On the convergence of shock-capturing streamline diffusion finite element methods for hyperbolic conservation laws', Math. Comput., 47, 1-18 (1990).

8. G.M. Hulbert and R.J.R. Hughes, 'Space-time finite element formulation for incompressible viscous flows', Comput. Methods Appl. Mech. Eng., 84, 327-348 (1990).

9. F. Shakib and T.J.R. Hughes, 'A new finite element formulation for computational fluid dynamics: IX. Fourier analysis of space-time Galerkin/least-squares algorithms', Comp. Methods Appl. Mech. Eng., 87, 35-58 (1991).

10. L.P. Franca, S.L. Frey and T.J.R. Hughes, 'Stabilized finite element methods: I. Application to the advective-diffusive model', Comp. Methods Appl. Mech. Eng., 95, 253-276 (1992).

11. T.E. Tezduyar, M. Behr and J. Liou, 'A new strategy for finite element computations involving moving boundaries and interfaces - the DSD/ST procedure: I. The concept and the preliminary numerical tests', Comp. Methods Appl. Mech. Eng., 94, 339-351 (1992).

12. T.E. Tezduyar, M. Behr, S. Mittal and J. Liou, 'A new strategy for finite element computations involving moving boundaries and interfaces - the DSD/ST procedure: II. Computation of free-surface flows, two-liquid flows and flows with drifting cylinders', Comp. Methods Appl. Mech. Eng., 94, 353-371 (1992).

13. S.K. Aliabadi and T.E. Tezduyar, 'Space-time finite element computation of compressible flows involving moving boundaries and interfaces', Comput. Methods Appl. Mech. Eng., 107, 209-223 (1993).

14. S. Mittal and T.E. Tezduyar, 'Direct time-integration methods: stabilized space-time finite element formulation of incompressible flows', in M. Papadrakakis (ed.), Solving Large-scale Problems in Mechanics, Wiley, New York, 1993.

15. A. Masud and T.J.R. Hughes, 'A space-time Galerkin/least-squares finite element formulation of the NavierStokes equations for moving domain problems', Comput. Methods Appl. Eng., 146, 91-126 (1997).

16. O.C. Zienkiewicz and R.L. Taylor, The Finite Element Method, vol. 1, McGraw-Hill, New York, 1989 (vol. 2, 1991).

17. C. Hirsch, Numerical Computations of Internal and External Flow, vol. 2, Wiley, New York, 1990.

18. A. Brooks and T.J.R. Hughes, 'Streamline upwind/Petrov-Galerkin formulation for convection dominated flows with particular emphasis on the incompressible Navier-Stokes equations', Comput. Methods Appl. Mech. Eng., 32, 199-259 (1982).

19. R. Codina, 'Comparison of some finite element methods for solving the diffusion-convection-reaction equation', Research Report No. 101, CIMNE, Barcelona, 1996.

20. O.C. Zienkiewicz and J.Z. Zhu, 'The superconvergent patch recovery (SPR) and adaptive finite element requirement', Comput. Methods Appl. Mech. Eng., 101, 207-224 (1992).

21. N.-E. Wibberg, F. Abdulwahab and X.D. Li, 'Error estimation and adaptive procedures based on superconvergent patch recovery (SPR)', Arch. Comput. Methods Eng., 4, 203-242 (1997).

22. F. Shakib, 'Finite element analysis of the compressible and Navier-Stokes equations', Ph.D. Thesis, Division of Applied Mechanics, Standford University, 1989. 